\title{
HOMBRES Y MUJERES INDÍGENAS: POBREZA Y RELACIONES DE GÉNERO EN MÉXICO
}

\section{INDIGENOUS MEN AND WOMEN: POVERTY AND GENDER RELATIONS IN MEXICO}

Juan Bello Domínguez. Universidad Nacional Autónoma de México y Universidad Pedagógica Nacional - México

juanbell@hotmail.com

Resumen Es importante llamar la atención sobre la equidad de género en la democratización de las sociedades contemporáneas. Las sociedades constituidas a partir de la multiculturalidad evidenciaron la falta de claridad de sus políticas sobre igualdad y equidad de género, en las que los pueblos indígenas inciden históricamente en el debate sobre las políticas públicas y las instituciones. El objetivo principal del presente artículo, es presentar el debate en torno a los referentes conceptuales, condiciones socioeconómicas y culturales de los hombres y mujeres de los pueblos indígenas y su permanente demanda de igualdad y equidad en el marco de la sociedad mundial y nacional. La estructura de las demandas y condiciones históricas de los hombres y mujeres de los pueblos indígenas, permite a lo largo de la investigación, establecer metodológicamente su relación histórica, conceptual y epistémica con la perspectiva de género. Incorporar el análisis de las características socioeconómicas y culturales de hombres y mujeres en la participación de los pueblos indígenas, es revisar sus condiciones en torno al desarrollo, reflexionar sobre su participación en las esferas económica, política, social y cultural, y con ello, gestar una critica de la política de la diversidad, igualdad y equidad de género en México.

Palabras clave Relaciones de Género, Pueblos Indígenas, Pobreza, Discriminación, México.

Abstract It is important to draw attention to gender equity in the process of democratization of contemporary societies. Societies formed from multiculturalism have evidenced the lack of clarity of their policies on gender equality and equity, in which indigenous people have historically influenced as actors in the debate on public policies and on the fate of institutions. The main objective of this article is to present a debate on the conceptual referents, the socio-economic and cultural conditions of the men and women of the indigenous communities and their permanent demand for equality and equity, within the framework of the world and national society. The structure of the demands and historical conditions of the men and women of the indigenous communities, allows throughout the investigation, to methodologically establish their historical, conceptual and epistemic relationship with the gender perspective. To 
incorporate the analysis of the socioeconomic and cultural characteristics of men and women in the participation of indigenous communities, is to review their conditions regarding on development, think about their participation in the economic, political, social and cultural spheres, and thereby develop a critique of the politics of diversity, equality and gender equity in Mexico.

Key words Gender Relations, Indigenous People, Poverty, Discrimination, Mexico.

\section{Sociedades diversas y relaciones de género}

Sólo en un Estado en el que la participación ciudadana sea posible, se pueden afrontar cuestiones tan urgentes como el reconocimiento de la diversidad cultural y la equidad de género, en la tarea de profundizar en la democracia y asegurar y garantizar la igualdad y justicia social.

En las últimas décadas aumentó la preocupación nacional e internacional por la identidad, equidad y los derechos de los pueblos indígenas (ONU, 2007). Además, la comunidad internacional expresó su determinación de atacar los altos niveles de pobreza y extrema pobreza, sobre todo en las áreas rurales. La correlación contundente que existe entre etnicidad y pobreza, en donde los pueblos indígenas, mayoritariamente, se encuentran de una manera desproporcionada entre los sectores mas pobres de la sociedad, ha hecho que se preste mayor atención al tema de los pueblos indígenas, la reducción de la pobreza y el desarrollo (ONU, 2015).

Un Estado democrático tiene obligación política y moral de atender también a estos colectivos minoritarios, trabajo que tiene dos dimensiones: una, la de lograr que los grupos mayoritarios y hegemónicos, comprendan a los "otros", los acepten, integren y asuman sus deberes de justicia y solidaridad para con ellos; otra, la de ayudar a estos grupos a no renunciar a su identidad, ni abandonar sus referentes culturales, para poder gozar de los mismos derechos y deberes de ciudadanía. Estos pueblos, necesitan tener garantizado el acceso a todos los servicios de que dispone la sociedad; los recursos para socializarse y crecer sin perder su identidad como miembros de sus pueblos de origen y de la nación (Bello, 2013).

En torno a las reformas para los pueblos indígenas, la equiparación entre cultura, lengua y/o grupo étnico correspondiente, es a todas luces, un error que no resiste argumentación alguna. Esto se traduce en una contribución a la tendencia a estereotipar a los individuos, de acuerdo con sus identidades étnicas o la nacionalidad que indica su pasaporte o la lengua que hablan sus padres en su casa, o sus abuelos en los respectivos lugares de origen. No se trata de defender la creencia, por otra parte infundada, de que todos esos factores (lengua, origen geográfico, identidad, etc.) no contribuyen a la construcción cultural. La idea que defendemos es la de no reducir la cultura a uno solo de 
esos factores o a la simple suma de todos ellos.

Contrariamente a lo que podría pensarse, la inmensa mayoría de los países son plurilingües, es decir, en su seno se hablan dos o más lenguas. El plurilingüismo es, por consiguiente, la norma y el monolingüismo la excepción. Son factores lingüísticos, sociales y políticos los que determinan, en un contexto dado, las diversas situaciones posibles entre las lenguas (UNESCO, 2003).

Las lenguas minoritarias se definen por los derechos sociales (más que por el tamaño del grupo lingüístico), o sea, por su falta o desigual equiparación a las mayoritarias, si bien, desde el prisma lingüístico, son susceptibles de abarcar toda la gama de situaciones posibles: variedades, dialectos, lenguas estandarizadas y cultas (CEPAL, 2017).

Los pueblos indígenas no pueden permanecer al margen del desarrollo, es el momento de generar mecanismos que permitan la convivencia entre todos los grupos que conforman la sociedad, teniendo como base su reconocimiento y respeto a su diferencia. A partir de la segunda mitad del siglo pasado, sus demandas son reconocidas por organizaciones internacionales y han sido motivo de modificaciones a las constituciones nacionales. Sin embargo, la denuncia permanente de los grupos marginados en forma general y las agresiones socioculturales y educativas a las comunidades indígenas, en particular, se fueron dando en forma creciente en diversos foros internacionales que retomaban, modificaban y actualizaban algunos acuerdos surgidos en años anteriores (Clavero, 1994).

La constatación de que existe una multiplicidad de pueblos diferentes, cada uno de los cuales, es portador de una cultura distintiva; ya no se ve como un lastre que debe eliminarse, ni como un obstáculo a vencer, sino por el contrario, se reconoce que la diversidad étnica y cultural, puede ser un recurso potencial en la educación, de enorme valía. Lo que aquí conviene destacar, es que ya no se plantea solamente tomar en cuenta la opinión y las aspiraciones de los pueblos indios y admitir su participación, sino afirmar que son ellos mismos, quienes deben tomar en sus manos las riendas de su proyecto educativo y cultural. Se les reconoce como sociedades culturalmente diferenciadas y, en consecuencia, legítimamente capaces de construir proyectos educativos autónomos dentro de los Estados nacionales de los que forman parte, por causa del devenir histórico.

Una de las principales reivindicaciones indígenas en las últimas décadas, se orienta al reconocimiento de sus derechos culturales y educativos en el plano nacional e internacional. Así, es patente una mayor presencia indígena en organismos internacionales, reflejada entre otros aspectos, en una creciente atención a sus demandas y en una más visible participación en la elaboración de normas, acciones y recomendaciones. Las reivindicaciones contienen una demanda de pluralismo social, 
cultural y educativo, y suponen, la superación definitiva del indigenismo tradicional, cuya crítica ya fue abiertamente planteada, convirtiendo ahora a las organizaciones indígenas, de meros intermediarios de sus problemas, en interlocutores directos (Sánchez, 1996).

La diversidad social, las relaciones de género y el desarrollo, son un proceso global donde están involucrados los ámbitos económico, político, social y cultural (UNESCO, 1996). La práctica ciudadana en torno al desarrollo y a las relaciones de género, es la participación en las esferas económica, política, social y cultural y con ello, necesariamente debe gestarse una política de la diferencia, en la que se reconozca la diversidad cultural y las demandas que genera, dando paso al pluralismo, a partir de la equidad de género, la participación y la organización de los diversos grupos sociales con prácticas sociales y culturales diferentes, en las que se contienen sus principios y conciencia de grupo, logrando legitimar el espacio como un espacio público de participación social (UNESCO,1996).

En el marco nacional de México, el término género alude a la distinción de los atributos de hombres y mujeres que son asignados por la sociedad. Las relaciones de género se expresan en valores, percepciones, prácticas, y actitudes sociales (INMUJER, 2004).

En el debate para el logro del proyecto de equidad de género, se plantea indispensable un escenario plural, de respeto a las diferencias y a la diversidad, de participación con equidad que conduzca a la discusión y al diálogo, y finalmente, consensos que tengan impacto en la justicia social para todas las personas indígenas. Diferentes grupos sociales, sufren la discriminación, marginación, opresión y exclusión. Situación que lleva a la institucionalización de la desigualdad y la diferencia basada en el principio de las culturas superiores y la hegemonía de un grupo. Al participar en esta confrontación, los grupos se ven insertos en ella, con mediaciones muy fuertes en que se acentúan a menudo las luchas por su autonomía y su identidad.

La emergencia de los sectores sociales y culturalmente diferenciados, tiene consecuencias importantes sobre la gestión de la vida social y de su transformación. El desarrollo comprendido como proceso de sustitución de prácticas y valores tradicionales, en torno a la construcción de un destino nacional homogéneo, es por definición, incompatible con el fortalecimiento de las identidades particulares y con el mantenimiento de la diversidad. El carácter multicultural que se reconoce ahora en la estructura nacional, demanda una nueva concepción del desarrollo y de la equidad de género, como un impulso desde las culturas, los intereses y las maneras de hacer de las comunidades; una redefinición de los roles de los diferentes actores en los escenarios locales, nacionales e internacionales, así como, condición de seguridad sobre los recursos, democracia en las decisiones y justicia en la distribución (CEPAL, 2017). 
La gestión de las transformaciones sociales no puede ignorar -- como lo hizo antes --, la presencia de actores organizados en torno a la recuperación y fortalecimiento de sus identidades culturales y de género, no puede desestimar la legitimidad de sus reivindicaciones, ni despreciar la dinámica de sus estructuras organizativas. Los pueblos indígenas han alcanzado un lugar en el escenario y han dicho su palabra; las respuestas hasta ahora no han establecido el diálogo, porque se procesan como concesiones o reacciones ante la emergencia desde instancias gubernamentales e institucionales, no concebidas como interlocutoras.

\section{La memoria histórica de hombres y mujeres indígenas. Hacia la Declaración de los Derechos de los Pueblos Indígenas}

A partir de la segunda mitad del siglo pasado, los pueblos indígenas y sus demandas, son reconocidas por organizaciones internacionales $y$ han sido motivo de modificaciones a las constituciones nacionales. Sin embargo, el incremento de la denuncia permanente de los grupos marginados en forma general y las agresiones socioculturales y educativas a las comunidades indígenas, en particular, se fueron dando en forma creciente en diversos foros internacionales que retomaban, modificaban y actualizaban algunos acuerdos surgidos en años anteriores (Clavero, 1994).

Uno de los logros de las últimas décadas en la lucha política de los pueblos indígenas, señala Magdalena Gómez (1997), ha sido el reconocimiento y aceptación del pluralismo étnico en el seno de las constituciones y los proyectos educativos nacionales. La influencia del movimiento indígena, en la progresiva aceptación de un mayor pluralismo por parte del Estado y de la sociedad, es indiscutible, desde que empezó a cobrar fuerza en los años setenta y se expandió y fortaleció en los ochenta. Esta progresiva toma de conciencia supone, en primer lugar, la constatación de la dimensión cuantitativa de la población y las políticas de reconocimiento impulsadas por los organismos multilaterales internacionales.

Lo que aquí conviene destacar, es que ya no se plantea solamente tomar en cuenta la opinión y las aspiraciones de los pueblos indígenas y admitir su participación, sino afirmar, que son ellos mismos, quienes deben tomar en sus manos las riendas de su proyecto social y cultural. Se les reconoce como sociedades culturalmente diferenciadas $y$, en consecuencia, legítimamente capaces de construir proyectos autónomos. Sin embargo, las condiciones socioeconómicas de estos pueblos, están registradas entre los indicadores de mayor marginación y exclusión en el mundo.

Kate Gilmore (Alta Comisionada adjunta para los Derechos Humanos de la ONU), declaró ante el Consejo de Derechos Humanos que:

Los 370 millones de indígenas, repartidos por 70 países, son el vivo ejemplo de las personas más desamparadas, marginadas y olvidadas del mundo (...) recordó 
que el $5 \%$ de la población mundial es indígena, pero que más de un tercio de la población más pobre pertenece a estos pueblos. Los que más se han quedado atrás, son los indígenas, representan más del 30\% de la pobreza extrema mundial. (ONU, 2018)

La Organización Internacional del Trabajo (OIT) en 1989, consensó el Convenio 169 en su Conferencia General congregada en Ginebra, el 7 junio 1989, en su septuagésima sexta reunión, lo que constituiría un avance importante del sistema jurídico internacional, en materia de derechos indígenas en lo general y, educativo en lo particular. Es importante destacar, su reivindicación incorporada al nuevo convenio de la OIT de 1989, sobre, protección de los pueblos indígenas y tribales.

El Convenio 169, se convirtió en una nueva versión del Convenio 107 de la Reunión de Expertos para la Revisión del Convenio sobre Poblaciones Indígenas y Tribales en su cuadragésima reunión, a la que asistieron representantes de la ONU, Organización Mundial de Salud, la UNESCO, el Banco Mundial y el Instituto Indigenista Interamericano.

El Convenio 169 de la OIT (OIT/INI, 2001), al incorporar las demandas indígenas y convertirlas en derechos universalmente reconocidos, sería el instrumento jurídico internacional más importante, para regularlos desde diferentes ámbitos de interés y como resultado de diversas consultas iniciadas desde 1986, no sólo con gobiernos y organizaciones no gubernamentales, sino también, con la participación de los mismos Pueblos Indígenas.

Abordar el problema de la ética, la educación y la cultura a partir de los acuerdos internacionales, nos invita a revisar el Convenio 169, que en su artículo 5 señala:

Al aplicar las disposiciones del presente Convenio: a) deberán reconocerse y protegerse los valores y prácticas sociales, culturales, religiosos y espirituales propios de dichos pueblos y deberá tomarse debidamente en consideración la índole de los problemas que se les plantean tanto colectiva como individualmente; $b$ ) deberá respetarse la integridad de los valores, prácticas e instituciones de esos pueblos; c) deberán adaptarse, con la participación y cooperación de los pueblos interesados, medidas encaminadas a allanar las dificultades que experimenten dichos pueblos al afrontar nuevas condiciones de vida y de trabajo (OIT/INI, 2001, p.8).

Para la atención de lo antes mencionado, el Convenio 169 señala en su artículo 7, el mejoramiento de las condiciones de vida y de trabajo y del nivel de salud y educación de los pueblos interesados, con su participación y cooperación, deberá ser prioritario en los planes de desarrollo económico global de las regiones donde habitan.

En su parte VI, el Convenio destaca el uso de la Educación y Medios de Comunicación para adaptar medidas y garantizar a los miembros de los pueblos interesados la 
posibilidad de adquirir una educación a todos los niveles, por lo menos, en pie de igualdad con el resto de la comunidad nacional.

Los programas y los servicios destinados a los pueblos interesados, deberán desarrollarse y aplicarse en cooperación con éstos, a fin de responder a sus necesidades particulares y abarcar su historia, sus conocimientos y técnicas, sus sistemas de valores y todas sus demás aspiraciones sociales económicas y culturales. Además, los gobiernos deberán reconocer el derecho de esos pueblos a crear sus propias instituciones, siempre que tales instituciones, satisfagan las normas mínimas establecidas por la autoridad competente en consulta con esos pueblos.

El artículo 28 del Convenio señala en forma general, adoptar medidas acordes a las tradiciones y culturas de los pueblos interesados, a fin de darles a conocer sus derechos y obligaciones, especialmente en lo que atañe al trabajo, a las posibilidades económicas, a las cuestiones de educación, salud y servicios sociales. Se adaptarán medidas de carácter sociocultural en todos los sectores de la comunidad nacional y especialmente en los que estén en contacto más directo con los pueblos interesados, con objeto de eliminar los prejuicios que pudieran tener con respecto a esos pueblos (OIT/INI, 2001).

Por otro lado, en la V Conferencia Iberoamericana de Educación (OEI, 1995), haciendo alusión a la Conferencia de Jomtien, Tailandia (UNESCO, 1990), se planteaban ideas respecto a los cuarenta años del derecho a la educación para todas las personas, en donde siguen las graves diferencias en el acceso a ésta. Asimismo, se reconoció que la educación puede contribuir a lograr un mundo más seguro, más sano, más próspero y ambientalmente más puro, y favorecer el progreso social, económico y cultural, la tolerancia y la cooperación internacional.

Al mismo tiempo y con la referencia al Plan de Acción de la Cumbre Social de Copenhague (ONU, 1995), se destaca que el componente vital en la lucha mundial contra la pobreza, debería ser la creación no sólo de empleo para todos, sino de empleos productivos que permitan a las personas salir y sacar a sus familias de la pobreza. Para ello, un objetivo importante debe ser: proteger la integridad de las culturas y los modos de vida autóctonos, así como, erradicar la discriminación en todas sus formas, promover la igualdad de oportunidades, la utilización de la educación como fuerza integradora y un mayor acercamiento entre el gobierno y el pueblo.

Por otro lado, el 25 y 26 de septiembre de 1997 en Mérida, Venezuela, se llevó a cabo la VII Conferencia Iberoamericana de Educación (OEI, 1997), con la temática "El Respeto y la Promoción del Pluralismo", cuyo planteamiento en su punto 3 señala:

La escuela debe ser capaz de formar personas que sepan desenvolverse y enfrentarse con éxito a la incertidumbre, que sepan tomar decisiones, que 
desarrollen satisfactoriamente sus competencias básicas y afiancen su identidad en la pluralidad. Los escenarios educativos que la escuela construye y/o acoge, tanto formales como informales, deben facilitar, además del aprendizaje individual, la interacción entre iguales, el aprendizaje cooperativo, el cultivo de la autonomía, el ejercicio del dialogo y el esfuerzo personal como entrenamiento para superar las dificultades y como aprendizaje para aquellas que puedan presentarse a lo largo de toda la vida. Para ello, debe integrar en su cotidianidad, y no sólo en los documentos legales que la regulan, valores propios de una comunidad democrática, equitativa y justa, basados en derechos y deberes de sus miembros para una convivencia respetuosa y feliz (OEI, 1997).

Asimismo, la Declaración de Sintra en la VIII Conferencia Iberoamericana de Educación (OEI, 1998), promovería las siguientes concepciones en su punto 4:

Desarrollar políticas orientadas a garantizar la igualdad de oportunidades y la calidad de las alternativas educativas, así como programas intersectoriales compensatorios, con atención prioritaria a los sectores más rezagados, sustentados en el principio de la equidad y orientados a la prevención de la exclusión social (OEI, 1998).

Para los fines de nuestro análisis, haremos referencia por último, a la IX Conferencia Iberoamericana de Educación (OEI, 1999), donde se postuló que con frecuencia coexisten, por una parte, el dinamismo de un sector de la población que avanza aceleradamente hacia una sociedad basada en el conocimiento y la información y, por la otra, grupos de personas analfabetas de adultos, que nunca cursaron o no terminaron su instrucción básica obligatoria y de niños, niñas y jóvenes que permanecen al margen de los servicios educativos.

Numerosos habitantes de la región --los grupos indígenas entre ellos--, se localizan en las zonas más inhóspitas, aisladas y dispersas. Con frecuencia, todavía carecen de servicios educativos, o la atención que reciben es insuficiente o inadecuada para responder a sus necesidades y aspiraciones. Paradójica y lamentablemente, tales carencias son experimentadas también en los sectores marginales de nuestras ciudades. Por motivos de diverso orden --históricos y culturales, de disponibilidad de recursos y dificultades de acceso, entre otros--, quienes más requieren de servicios educativos de calidad, son quienes tienen un acceso más restringido a ellos.

La equidad no se limita solamente al acceso universal a los servicios, sino que requiere de una atención diferenciada, que responda a la variedad de necesidades que presentan cotidianamente los hombres y mujeres indígenas.

Por otra parte, en el Proyecto de Declaración sobre los Derechos de los Pueblos Indígenas de la ONU (ONU, 1995), se advierten modificaciones sustanciales, que representarían un reconocimiento importante para la consolidación del respeto a la diferencia. Se reconocen 
los derechos fundamentales en materia social, cultural y educativa de los pueblos indígenas; el goce de sus libertades y el respeto a sus valores; el derecho a decidir sus prioridades y el control sobre su desarrollo; el derecho a mantener y practicar sus costumbres y, el reconocimiento de la importancia de conservar y promover su idioma.

Se colocaría al ser humano como centro, sujeto y actor del desarrollo, con la finalidad de lograr un desarrollo integral, que conduzca a la realización plena de la capacidad creativa y productiva, elevando su calidad de vida, con la pretensión de superar la pobreza y teniendo presente el respeto a los derechos civiles y humanos, por lo tanto, a la vida en todas sus manifestaciones. Así, el desarrollo debe considerar no sólo la calidad de vida, sino también, el respeto a los distintos estilos de vida (UNESCO, 1996).

El creciente interés de la opinión pública en las poblaciones indígenas y un largo proceso de negociaciones internacionales, en el que participaron las organizaciones indígenas, indujeron a la comunidad internacional a proclamar el año de 1993, el Año Internacional de las Poblaciones Indígenas del Mundo, y, posteriormente, al período 19942003, Decenio Internacional de las Poblaciones Indígenas del Mundo.

Además, desde 1995 se celebra el 9 de agosto, el Día Internacional de las Poblaciones Indígenas del Mundo. Cada una de estas medidas, ha tenido importancia en la lucha por el reconocimiento de los derechos de las poblaciones indígenas, medidas que cobran mayor significación con el documento histórico relacionado con los derechos humanos y aprobado por las Naciones Unidas hace más de setenta años: la Declaración Universal de Derechos Humanos (ONU, 1948). Se reconoce prioritariamente la dignidad inherente a todo ser humano y se establecen los derechos de que deben disfrutar todos, sin distinción de ningún tipo por motivo de raza, color, sexo, idioma, religión, convicciones políticas, o de otra índole, origen nacional o social, propiedad, nacimiento u otra condición.

Existen pocas leyes, que consagren los derechos fundamentales de los Pueblos Indígenas. En el ámbito internacional, algunos países no han ratificado los instrumentos jurídicos en esta materia. Habría que tomar en cuenta, que se presentan problemas al momento de implementar las normas jurídicas; es decir, el espíritu de la ley establece una situación jurídica distinta a la que se produce en la realidad. Adicionalmente, se debe enfatizar que los derechos indígenas, no pueden ser interpretados solamente a la luz del derecho común. En diversos países, se aplica una interpretación distinta a la visión de estos pueblos. De igual forma, en reiteradas ocasiones dentro del proceso de formación de las leyes, no se toma en cuenta su punto de vista.

La celebración del cincuentenario de la Declaración Universal de Derechos Humanos, constituyó una oportunidad especial para que la comunidad internacional, reflexionara acerca de los progresos alcanzados en el mejoramiento de las vidas de los desposeídos y 
se adoptaran medidas eficaces, para enfrentar los desafíos que aún quedan por delante. Se podrían realizar esfuerzos especiales para aumentar el bienestar de las poblaciones indígenas y convertir los derechos básicos y las libertades fundamentales proclamados en ella, para el disfrute de toda la humanidad. En este sentido, la universalidad, indivisibilidad e interdependencia de los derechos humanos cobran importancia capital.

Después de más de 20 años de lucha permanente en el contexto internacional y en el seno de la Organización de la Naciones Unidas, sería aprobada la Declaración de las Naciones Unidas sobre los Derechos de los Pueblos Indígenas, por su Asamblea General el 13 de septiembre de 2007 (ONU, 2007). Se reconocen los derechos humanos básicos y las libertades fundamentales de las poblaciones indígenas y se pide a los Estados que respeten y acaten cualquier instrumento jurídico convenido entre ambas partes. En el proyecto de declaración, se hace hincapié en el derecho de las poblaciones indígenas a ser libres y a disfrutar de igualdad con todas las demás personas y poblaciones en dignidad y con derechos; el derecho a la libre determinación y a decidir libremente su estatuto político y su desarrollo económico, social y cultural; el derecho a practicar y revitalizar sus tradiciones culturales y sus costumbres, y también el derecho a cultivar y enseñar sus tradiciones espirituales y religiosas; el derecho a establecer y supervisar sus sistemas de enseñanza y las instituciones que proporcionan instrucción en sus propios idiomas; el derecho a participar plenamente en todos los niveles de adopción de decisiones, en cuestiones que puedan afectar a sus derechos, sus vidas y su destino; el derecho de las poblaciones indígenas, a sus tierras, territorios y recursos (ONU, 2007).

En el marco global, los gobiernos nacionales han comenzado a normar el respeto a la diferencia en la igualdad, la necesidad de poner en práctica no sólo políticas educativas y culturales diferenciadas para los pueblos indígenas, sino que, éstas se elaboren cada vez más con su colaboración y asentimiento; y que en este caso, como en todo análisis de situación de la problemática indígena, sea preciso mantener siempre una doble perspectiva: la que corresponde a su propia visión interna y aquella procedente de agentes externos. A partir de la Declaración en 2007, se lograron avances significativos en varios asuntos que competen a su calidad de vida. No obstante, estos avances son aún insuficientes, habida cuenta de los efectos de siglos de discriminación. La deuda sigue en considerar la equidad en la perspectiva de genero, entre los hombres y mujeres en el contexto nacional e indígena (CEPAL, 2017).

\section{La equidad de género, entre la marginación y pobreza de los hombres y mujeres indígenas de México}


El desarrollo social de México, se desplegó en medio de profundas asimetrías en el desarrollo regional. A lo largo del siglo pasado, unas cuantas entidades fueron el escenario privilegiado del proceso de modernización. En cambio, en las entidades donde la modernización fue limitada, se fueron acumulando los rezagos sociales de hombres y mujeres, aumentando con ello, la brecha de desarrollo que las separaba de las regiones más avanzadas. El patrón de asentamientos humanos del país, se caracterizaría por una fuerte concentración de población en unos cuantos centros urbanos y una acentuada dispersión en numerosas y pequeñas localidades en todo el territorio (Adler y Flores, 2002).

La desigualdad en el interior de los municipios y las menores oportunidades de participación de los hombre y mujeres en los asentamientos situados en la periferia, genera que los problemas se agudicen al no contar con la infraestructura, equipamiento, demanda de trabajo y oferta de servicios básicos suficientes y contribuya a generar corrientes migratorias hacia las localidades más consolidadas o centros urbanos nacionales o extranjeros. Con ello, la marginación y pobreza de la población se reproduce en el interior de los municipios: los municipios más desarrollados reciben más; los municipios eminentemente rurales, se ven perjudicados cuando se emplea el criterio del peso demográfico y la cobertura es insuficiente de los programas gubernamentales especialmente los dirigidos a grupos indígenas y marginados. Algunos estados optan por destinar los recursos que reciben a las zonas urbanas, abandonando a los pequeños poblados y al campo y con ello, se atenta contra la equidad de género entre las comunidades indígenas. En el contexto del México actual y la caracterización de la pobreza y las relaciones de género entre las comunidades indígenas, en las que destacan las entidades que se encuentran en el rango de marginación alto y muy alto. Identificamos que son las que registran los mayores indicadores de marginación y no necesariamente las que concentran el mayor número de hablantes de la lengua indígena (criterio mayoritariamente usado en otras investigaciones) con el propósito de reconocer las características sociales económicas, culturales, y educativas de los hombres y las mujeres indígenas en México (Bello, 2007).

Las diferencias por sexo en la participación económica se presentan prácticamente en toda sociedad, los pueblos indígenas no son la excepción, hay diferencias tanto en el grado de participación como en el tipo de actividades que desarrollan. La participación de la mujer en el ámbito indígena se presenta con un crecimiento importante y la condición económica de la población remunerada, es precaria.

Cierto es que el espacio natural de trabajo preponderantemente para la población indígena, ha sido por siglos la agricultura; actividad indudablemente noble pero abandonada por la política económica nacional durante varias décadas, provocando baja 
rentabilidad al extraerle sus excedentes y limitando así su inversión.

En lo que se refiere la infraestructura social básica en las comunidades indígenas del país, se registra en 2015 que del total de viviendas, el $12.8 \%$ no cuenta con agua potable; el $26.9 \%$ no tiene drenaje, el $4.4 \%$ no cuenta con energía eléctrica, $13.9 \%$ cuenta con piso de tierra y por último, 58.8\% de viviendas en las que se cocina con leña (INEGI, 2015).

La problemática de salud pública en las comunidades indígenas, registrada con algunos indicadores en 2015, se expresa de la siguiente manera con la distribución porcentual de la población indígena, sin derechos a los servicios de salud por sexo: a) mujeres $45.7 \%$ b) hombres 54.3\% (INEGI, 2015).

Los logros de la modernización de la educación indígena y de la equidad de género son relativos, pues persisten muchos de los grandes problemas, como son las profundas desigualdades socioeconómicas, la marginación social, la miseria extrema y un enorme rezago educativo. Tampoco se ha logrado cubrir la totalidad de las lenguas que se hablan en el país, aludiendo que el número de hablantes de algunas de ellas no es significativo (UPN/INEE, 2016).

No se puede negar que la cobertura a esta población, se ha ampliado - como lo manifiesta el Instituto Nacional para la Evaluación de la Educación (INEE, 2017) --, sin embargo, no se ha alterado la grave problemática que afecta a la educación para los indígenas, dado que se mantienen índices altos de reprobación, deserción, y una baja eficiencia terminal; asimismo prevalecen indicadores de pobreza y marginación.

Es preciso tener en mente que la igualdad de oportunidades no solo depende de la oferta educativa. Algunos de los factores que dan origen y sustentan la inequidad en la educación por motivos de género, se relacionan con otros cuya semilla y abono se encuentra en las relaciones familiares, en las percepciones de los roles de hombres y mujeres y en las expectativas y prácticas cotidianas dentro de los hogares y las comunidades, es decir, en la cultura.

Distribución porcentual de la Población indígena mayor de 15 años que no sabe leer ni escribir un recado, por sexo en el 2015: 65\% eran mujeres y 35\% hombres. La distribución porcentual de la población indígena de 6 a 14 años y más, que no asiste un centro de enseñanza del sistema educativo, por sexo: $50.7 \%$ son mujeres y 49.3 son hombres. Por otro lado, la distribución porcentual de la población indígena mayor de 15 años sin instrucción escolar, por sexo: mujeres $63.7 \%$ y hombres $36.3 \%$ (INEGI, 2015).

Tampoco se ha logrado cubrir la totalidad de lenguas que se hablan en el país, aludiendo que el número de hablantes de los grupos cuantitativamente más pequeños, no es significativo y el llevarles escuela implicaría un derroche de recursos económico y humanos; (muchos de los niños que no reciben servicios educativos, viven en localidades 
que tienen menos de 100 habitantes); en este sentido, tenemos la presencia de la política focalizadora, al seleccionar las lenguas y entidades a atender, y en la elaboración de materiales, que sólo se enfocan a la enseñanza de la lengua, y no se abarcan las demás asignaturas que conforman el plan de estudios (INEE, 2017).

\section{Mujeres e identidad indígena}

La participación de las mujeres indígenas en los procesos con prácticas sociales y culturales diferentes, engendra conciencia de grupo e identidad social en un espacio público de participación social respecto a los otros (Bello, 2013).

El debate de la participación de las mujeres indígenas se plantea como un escenario plural y de consolidación de sus diferencias culturales, pero sobre todo, de una participación que conduce a la discusión, al diálogo y a los consensos que impactan en su identidad grupal.

Es importante llamar la atención sobre la participación creciente de las mujeres indígenas en torno a la defensa de su diversidad cultural y social, porque es un signo frente a comunidades diferentes de las suyas, porque durante los procesos generados por la movilidad y el contacto con otras formas de concebir al ser humano y a la naturaleza, son ellas quienes promueven el tránsito de los valores sociales, comunitarios, étnicos y lingüísticos propios. Negar la tensión que provoca este encuentro de los indígenas y la participación de sus mujeres, es negar las tensiones entre la diversidad y la homogenización (Quintero y Fonseca, 2008).

Las comunidades constituidas a partir de la multiculturalidad y de la multietnicidad, han evidenciado la falta de claridad en las políticas gubernamentales sobre lo diverso. Los sectores cultural y étnicamente diferenciados en constante movimiento, han conquistado un papel protagónico en el debate sobre las políticas públicas y sobre el destino de las instituciones (Sánchez, 1996).

La lucha por el reconocimiento de los derechos colectivos en poblaciones indígenas, se desprende en gran medida, de la especificidad cultural que las mujeres indígenas han defendido y construido en el intercambio con otros pueblos, etnias, regiones y comunidades, constituyéndose ellas en el eje de respeto a su identidad étnica y cultural, y el reclamo a políticas públicas que atiendan sus demandas identitarias.

La estructuración de las demandas de estos grupos, como alternativas posibles, y su nivel de crecimiento en torno a la organización de su cultura con la participación de las mujeres, permitirán a éstos, insertarse en los escenarios nacionales e internacionales en condiciones socioculturales de mayor equidad (Clavero, 1994). Los movimientos indígenas incrementados durante los últimos años, con la participación de la mujer y su 
demanda de reconocimiento de la diversidad cultural y étnica, provocaron respuestas en los ámbitos internacional y nacional; los gobiernos, las instituciones y la sociedad en general, se vieron obligados a ampliar los espacios para el desarrollo, formación y consolidación de la diversidad como característica prioritaria en la región de América Latina. Una de las expresiones más claras de estos procesos de profundización, materialización y objetivación de la diversidad, son los cambios que en materia de tradición constitucional y legal promovieron el surgimiento, desarrollo y consolidación de los movimientos indígenas (Gómez, 1997).

Con frecuencia, los indígenas son considerados como campesinos y los campesinos como indígenas, mientras que la especificidad del problema desaparece, o el indígena es aislado del resto del pueblo, de los trabajadores rurales y de los pobladores urbanos. Al mismo tiempo, las circunstancias bajo las cuales el indígena se convierte en campesino o en trabajador agrícola, nunca se establecen claramente. Otra posibilidad para definirlos es usar las lenguas y dialectos como un indicador, a fin de saber quién es. Pero la definición real de indígena tiene dos significados: uno, relacionado con el sistema global en que los indios viven y trabajan, y otro, con las diversas culturas, lenguas y organizaciones políticas de las comunidades (Bello, 2007).

El movimiento de los pueblos indígenas y la participación de las mujeres, refiere algunas contradicciones que provocan una crisis en el esfuerzo por construir una plataforma de análisis común, tejer una red conceptual amplia para la conquista de espacios sociales, jurídicos y culturales, inicia con un proceso de agregación de las demandas particulares y diversas de los indígenas en los ámbitos local y regional y supone, la generación de nuevas categorías que reivindican su autonomía en torno a su identidad y a su pueblo.

El desarrollo económico y social de los indígenas, entendido como proceso de sustitución de prácticas y valores tradicionales en torno a la construcción de un destino de homogenización, es por definición, incompatible con el fortalecimiento de las identidades particulares y con el mantenimiento de la diversidad. El carácter multiétnico y multicultural de movimientos indígenas, que se reconoce ahora con la participación de las mujeres indígenas, demanda una nueva concepción del desarrollo de comunidades, como un impulso desde las culturas, los intereses y las maneras de hacer de las comunidades; una redefinición de los roles de los diferentes actores en los escenarios locales, nacionales e internacionales (Castro y Casique, 2008).

La gestión de las transformaciones sociales en procesos socioculturales no puede ignorar, como lo hizo antes, la presencia de las mujeres indígenas en torno a la recuperación y fortalecimiento de sus identidades étnicas y culturales, ni tampoco desestimar la legitimidad de sus reivindicaciones ni despreciar la dinámica de sus 
estructuras organizativas. El paso a la visión pluricultural, tiene una primera condición fundamental: el diálogo interétnico e intercultural. Las mujeres indígenas han alcanzado un lugar en el escenario sociocultural y han dicho su palabra; las respuestas institucionales hasta ahora no han establecido el diálogo, porque se procesan como concesiones o reacciones ante la emergencia, desde instancias gubernamentales e institucionales que no son concebidas como interlocutoras (Careaga y Jiménez, 2011).

En el problema que nos interesa de la participación de las mujeres indígenas y el de los criterios definitorios del concepto de etnia, la cultura como sistema de valores y significados, tiene una importancia indudable que no puede desvincularse de los fenómenos de estructura que subyacen a ella. En la medida en que la cultura es factor dinámico y sujeto a cambios por múltiples razones, los cambios culturales condicionan también la identidad y viabilidad de los grupos étnicos.

La organización social e identitaria en procesos migratorios es aquel conjunto de relaciones sociales que da consistencia a un grupo étnico, más allá de la identidad de cada uno de sus miembros. En la medida en que los miembros de una etnia participan en la organización social de su grupo promovida por sus mujeres, aumenta su dependencia con respecto al grupo y a sus valores colectivos. La organización social establece los límites del grupo, constituye el marco de referencia para distinguir el nosotros del ellos, los propios y los ajenos, los miembros y los extraños (CEPAL, 2017).

En su sentido amplio, la cultura constituye un conjunto de elementos distintivos de las etnias: sistemas de valores, símbolos, significados, normas y costumbres, que distinguen a los de adentro, de los de afuera. En el terreno de la migración se ha transformado la circulación de lenguajes, mensajes y productos. El sistema comunicativo promovido por las mujeres indígenas en movilidad territorial, tiene como misión la reorganización de la vida cotidiana y los saberes (Castro y Casique, 2008).

Los límites entre las culturas son flexibles y cambiantes. La movilidad de los migrantes indígenas encuentra una diversidad de culturas de diferentes grupos sociales, aun cuando se comparten rasgos comunes de cultura al interior.

El análisis de la relación jerarquizada y desigual de los movimientos indígenas conduce al enfrentamiento de los ámbitos local, regional y nacional, en torno a una visión que pretende erigirse como única, en donde el ámbito nacional supone ser la síntesis del local y del regional, como unidades subordinadas, situación que automáticamente se hace extensiva a los grupos e individuos que los conforman.

La participación de la mujeres indígenas en la integración de las organizaciones del conjunto del pueblo, parece ser de los pocos caminos que permiten comprender los problemas del indio en su especificidad; como parte de los problemas de los grupos en 
permanente movilidad y de un programa de acción común de un pueblo, unido y respetuoso de las diferencias y colectividades.

La memoria colectiva engendrada por las mujeres indígenas en movimiento territorial, puede entenderse como un ancla de la identidad $y$, a la vez, como un vasto campo fértil para el surgimiento de nuevas y variadas formulaciones de la identidad. Así, las identidades colectivas no existen en forma pura y fija, sino que se forjan a partir de una multiplicidad de elementos interrelacionados, susceptibles de modificación en el curso del tiempo y del espacio que ocupan los indígenas y sus mujeres (Careaga y Jiménez, 2011).

Con base en estos argumentos, saber cómo los pueblos indios pueden vincularse a contextos locales, regionales, nacionales e internacionales, sin perder su identidad. La participación de las mujeres indígenas es una importante categoría social y cultural, aunque manifiesta serios obstáculos para expresarse como categoría política. Las diferencias de intereses estrechos dividen constantemente a los miembros de la misma cultura y de la misma condición socioeconómica y cultural. La participación de la mujer indígena muestra en la mayoría de los casos, un sentido particularista de pertenencia; como una autoafirmación que se refuerza a través de luchas constantes por la reivindicación de su identidad (CEPAL, 2017).

\section{Conclusiones}

La perspectiva y argumentación del presente artículo, exigió una reflexión sobre la elaboración de políticas sociales, culturales y educativas dirigidas a las mujeres y hombres indígenas, no solamente vinculadas con el desarrollo económico, sino también aquellas, que contribuyan a enriquecer el ámbito de equidad y participación en los procesos de socialización, inclusión y cultura de estos pueblos.

Resulta indispensable que se establezcan políticas públicas que consideren la diversidad, cooperación cultural y desarrollo, emanadas de las recomendaciones de los principales organismos multilaterales. El surgimiento de organizaciones paralelas a las estructuras de los Estados, fortalecerá la cooperación local, nacional e internacional en torno a los pueblos indígenas, para trabajar lo global desde lo local y a su vez, fomentar el contacto y la conformación de redes culturales, favorecidas por las nuevas posibilidades de su desarrollo social, cultural y económico.

Veamos el mundo de los hombres y mujeres indígenas, como un escenario de diálogo en donde la multiplicidad de culturas no se subordina, sino que tratan de comprenderse en el plano de la relación intercultural. En el marco de las relaciones culturales de las mujeres, hombres y comunidades indígenas, se construirán nuevos centros de concertación, negociación y rupturas. Es así como las semejanzas, diferencias, 
discordancias y concordancias, constituirán nuevos ámbitos de interacción, donde el sentido de las prácticas colectivas se producen, reproducen y confrontan a partir de nuevos esquemas de negociación y disputa social, cultural y económica.

Los cambios implican una profunda redefinición de la participación de hombres y mujeres indígenas en el contexto comunitario y regional. Las identidades se construyen entre lo individual y lo social, dentro de un marco histórico y simbólico; las transformaciones en lo referente a las identidades, se insertan en prácticas cotidianas a través de la familia, el trabajo, las condiciones objetivas de vida o de la identificación con proyectos imaginarios, donde las personas se incorporan en comunidades de carácter religioso, generacional o étnico.

No basta con la formulación de políticas nacionales basadas en el respeto mutuo y la libertad cultural de los pueblos indígenas, se hace necesario el ejercicio de la relación dialógica comunitaria y regional, como base de la convivencia de la diversidad entre las colectividades, en el marco del Estado sustentado en un compromiso ético. En torno a la diversidad cultural, los hombres y mujeres indígenas han luchado por encontrar espacios para permanecer, manifestarse y ser escuchados, para acceder al diálogo; espacios negados, pero que les permiten definirse, movilizarse, afirmar su cultura, su identidad y dar sentido a su vida, sobre todo, en constantes movimientos.

\section{Bibliografía}

Adler, L. y Flores, J. (2002). "Los indígenas y el sector informal urbano" en Estado del Desarrollo Económico y Social de los Pueblos Indígenas de México. Segundo Informe, INI-PNUD (pp.111-113) México: Ed. Instituto Nacional Indigenista y Programa de Naciones Unidas para el Desarrollo.

Bello, J. (2007). Educación y Pueblos Excluidos. México: Ed. Miguel Ángel Porrúa/Cámara de Senadores/UNAM.

Bello, J. (2013). "Educación Intercultural. ¿Trabajar con los Diferentes o las Diferencias?" Revista Científica Ra Ximhai, 9 (1) Enero-Abril, pp.61-73

Careaga, G. y Jiménez, P. (2011) La feminización de la pobreza en México. (1 $1^{\mathrm{a}}$ ed) México: Ed. Cámara de Diputados. Comisión de Equidad y Género. LXI Legislatura.

Castro, R. y Casique, I. Estudio sobre cultura, género y violencia contra mujeres. México: UNAM/Centro Regional de Investigaciones Multidisciplinarias.

CEPAL (2017). Planes de igualdad de género en América Latina y el Caribe. Mapas de ruta para el desarrollo. Santiago de Chile: Comisión Económica para América Latina y el Caribe.

Clavero. B. (1994). Derecho indígena y cultura constitucional en América. . México: Siglo XXI Editores 
Gómez, M. (1997). "Derecho indígena y constitucionalidad: El caso Mexicano" en M. Gómez. Derecho Indígena. México: Ed. Instituto Nacional Indigenista.

http://www.acnur.org/fileadmin/Documentos/Publicaciones/2010/7375.pdf (Consultado el 31 de agosto del 2018).

INEE (2017). La educación obligatoria en México. Informe 2017. México: Instituto Nacional para la Evaluación de la Educación

INEGI (2015). Encuesta Intercensal en México, México: Ed. Instituto Nacional de Estadística, Geografía e Informática.

INMUJER (2004). El enfoque de género en la producción de estadísticas educativas en México. México: Ed. Instituto Nacional de las Mujeres.

OEI (1995). Conferencia Iberoamericana de Educación, Buenos Aires Argentina, 7 y 8 de septiembre de 1995. https://www.oei.es/historico/vcie.htm (Consultado 28 agosto de 2018)

OEI (1997). VII Conferencia Iberoamericana de Educación. Celebrada en Mérida, Venezuela, 25 y 26 de septiembre de 1997. https://www.oei.es/historico/viicie.htm Consultada en 29 de agosto de 2018).

OEI (1998). VIII Conferencia Iberoamericana de Educación. Celebrada en Sintra, Portugal, el 9 y 10 de julio de 1998. https://www.oei.es/historico/viiicie.htm Consultado el 29 de agosto de 2018.

OEI (1999). IX Conferencia Iberoamericana de Educación Celebrada en La Habana, Cuba 1 y 2 de julio de 1999. https://www.oei.es/historico/ixcie.htm Consultado el 29 de agosto de 2018.

OIT/INI (2001). Organización Internacional del Trabajo, Convenio 169. X aniversario de su vigencia en México. México: Instituto Nacional Indigenista.

ONU (1948) Declaración Universal de Derechos Humanos. http://www.un.org/es/universaldeclaration-human-rights/Consultado el 29 de agosto de 2018.

ONU (1995). Proyecto de Declaración sobre los Derechos de los Pueblos Indígenas de la ONU. $\quad$ https://www.ohchr.org/Documents/Publications/GuidelPleaflet5sp.pdf Consultado el 19 de agosto de 2018.

ONU (1995). Cumbre Social de Copenhague. ONU. http://www.un.org/documents/ga/conf166/aconf166-gsp.htm (Consultado el 20 de agosto de 2018).

ONU (2007). Declaración de las Naciones Unidas sobre los Derechos de los Pueblos Indígenas. https://undocs.org/es/A/RES/61/295. Consultado el 29 de agosto de 2018.

ONU (2015). Objetivos de Desarrollo del Milenio Informe de 2015. Nueva York, Estados Unidos: Organización de las Naciones Unidas. 
ONU. (2018). "Los indígenas siguen siendo las personas más marginadas del mundo" en Noticias ONU: https://news.un.org/es/story/2018/og/1441862. Consultado el 29 de noviembre de 2018.

Quintero, M. Y C. Fonseca (Coord)(2008). Investigaciones de Género. Aspectos Conceptuales y Metodológicos. México: Ed. Cámara de Diputados y Miguel Ángel Porrúa.

Sánchez. C. (1996). "Las demandas indígenas en América Latina y el derecho internacional" en P. González y Roitman, M. (Coord). Democracia y Estado Multiétnico en América Latina (pp.95-106) México: Ed. La Jornada EdicionesCentro de Investigaciones Interdisciplinarias en Ciencias y Humanidades/ UNAM.

UNESCO (1996). Nuestra diversidad creativa. Informe de la Comisión Mundial de Cultura y Desarrollo. México: UNESCO/Correo de la UNESCO.

UNESCO. (2003). La educación en un mundo plurilingüe, Paris, Francia: Correo de la UNESCO.

UPN/INEE (2016). Consulta Previa, Libre e Informada a Pueblos y Comunidades Indígenas sobre la Evaluación Educativa. Informe General de Resultados. México: Universidad Pedagógica Nacional e Instituto Nacional para la Evaluación de la Educación.

\section{Cómo referenciar este artículo/How to reference this article:}

Bello Domínguez, J. (2019). Hombres y mujeres indígenas: pobreza y relaciones de género en México. iQUAL. Revista de Género e Igualdad, 2, 114-132, doi: 10.6018/iQual.340291

Bello Domínguez, J. (2019). Hombres y mujeres indígenas: pobreza y relaciones de género en México. [Indigenous men and women: poverty and gender relations in Mexico]. iQUAL. Revista de Género e Igualdad, 2,114-132, doi: 10.6018/iQual.340291 